\title{
El rol de los pasos peatonales subterráneos como alternativa en los actuales esquemas de planeación urbana
}

\section{The role of underground pedestrian crossing as an alternative way to the current urban planning schemes}

\section{Carlos Felipe Urazán Bonells}

Ingeniero Civil, doctor en Gestión Territorial e Infraestructuras del Transporte. Docente Asociado de la Universidad de La Salle. Bogotá, Colombia.

Contacto:caurazan@unisalle.edu.co

\section{Adolfo Camilo Torres Prada}

Ingeniero Civil, doctor en Ciencias Técnicas. Docente Asociado de la Universidad de La Salle. Bogotá, Colombia. Contacto: atorresc@unisalle.edu.co

\section{Édgar Humberto Sánchez Cotte}

Ingeniero Civil, magíster en Ingeniería Civil. Docente Asistente de la Universidad Distrital Francisco José de Caldas. Bogotá, Colombia.

Contacto:esanchezc@udistrital.edu.co

Fecha de recepción: 30 de enero de 2013

Clasificación del artículo: reflexión

Fecha de aceptación: 15 de abril de 2013

Financiamiento:Universidad de La Salle

Palabras clave: infraestructura peatonal, movilidad urbana, túneles peatonales.

Key words: pedestrian infrastructure, urban mobility, pedestrian tunnels.

\section{RESUMEN}

El planteamiento de pasos peatonales subterráneos como solución infraestructural a la actual tendencia de la movilidad urbana, se sustenta en diversos criterios como: la tendencia que siguen algunas ciudades de países desarrollados en una mayor disposición de espacios subterráneos multifuncionales, la disminución del riesgo de acci- dentalidad vial respecto de los cruces peatonales a nivel, menores costos de construcción, menor ocupación del espacio público en los accesos, y mayores beneficios para el usuario respecto de los puentes peatonales: protección ante impactos ambientales negativos, menores distancias de recorrido y, por tanto, mayor velocidad de desplazamiento, lo cual reduce la percepción de mayor cansancio al usar las infraestructuras y un menor 
impacto visual negativo. No obstante los beneficios, la implantación de pasos peatonales a desnivel compiten con factores de localización, como es el hecho de ubicarse cerca de pasos semaforizados a nivel, lo cual modifica la intención del peatón de cómo va a cruzar la vía.

\section{ABSTRACT}

The underground pedestrian crossings approach has an infrastructural solution to the current urban mobility. It is based on various criteria such as: the tendency to follow the availability of multifunctional underground spaces of some cities in developed countries, which can reduce the risk of accidents on road at pedestrian crossings, lower construction costs, lower occupancy of public space. Also, the use of underground pedestrian crossing can benefits more the user compared with pedestrian bridge in the following aspects: Protection under negative environmental impacts and reducing more travel distances. As a consequence, this solution would reduce the perception of increased fatigue in the pedestrian using the infrastructure, and would have less negative visual impact. Despite the benefits, the implementation of pedestrian crossings underpass competing with location factors such as the fact of being located near steps at traffic lights, which change the intention of pedestrians on how to cross roads.

\section{INTRODUCCIÓN}

El actual desarrollo urbano que se vive en América Latina es altamente dependiente de los cambios en infraestructura vial. En los procesos de diseño de este tipo de infraestructura prima la condición de movilidad del peatón; y dentro de este tópico, los cruces viales juegan un papel fundamental en la seguridad vial y por tanto en la calidad de vida. Y para dar solución a los cruces viales de alto flujo suele optarse por pasos peatonales subterráneos o por pasos elevados.

Sin embargo, los planteamientos subterráneos vienen demostrando tener mayores ventajas para el peatón. Vale la pena contemplar las virtudes reconocidas de los túneles peatonales dentro del espacio urbano, tal como lo plantea Koniohov, los pasos peatonales en túnel presentan diversas ventajas frente a los puentes [1]. Adicionalmente, cabe recordar que la construcción de obras subterráneas resulta de las demandas de población, en búsqueda de una mejor calidad de vida, con formas más sencillas de llevar agua a las viviendas, almacenar comida, protegerse en la guerra y transportarse, entre otras necesidades que se mantienen en la actualidad, pero adicionándole a éstas el problema de la falta de espacio en superficie [2].

El presente artículo expone diferentes aspectos que dan soporte a una tendencia, cada vez mayor, a solucionar la condición peatonal urbana por medio de infraestructuras subterráneas; desde el punto de vista de la disposición de grandes ciudades, hasta la percepción misma del usuario.

\section{LOS ESPACIOS PEATONALES SUBTERRÁNEOS EN EL DESARROLLO URBANO}

En el presente, son varias las ciudades en el mundo que están propendiendo por planificar y desarrollar circulaciones peatonales subterráneas, con acceso a diversos servicios como: estacionamientos, estaciones de transporte público y galerías comerciales. Esa es la directriz que deben seguir las grandes y medianas urbes, tanto en países desarrollados, y en mayor grado, las ciudades de países en vía de desarrollo. 
El libro blanco del Comité de espacio subterráneo, de la Asociación Internacional de Túneles y Espacio Subterráneo [3] (ITACUS, pos sus siglas en inglés) expone que las actuales tasas de incremento poblacional en las ciudades, sumado a las exigencias por una mejor calidad de vida y condiciones ambientales, están generando una fuerte apuesta y demanda por los desarrollos urbanos subterráneos. Estos requerimientos han puesto de manifiesto los errores que tradicionalmente venían acompañando las obras subterráneas no planificadas: altos costos en relocalización de infraestructuras, falta de aprovechamiento de las mejores condiciones geológicas disponibles, y la visión de las redes de transporte cada vez a mayor profundidad para dar espacio a futuros desarrollos urbanos subterráneos.

Los errores mencionados pueden sintetizarse en que el uso del espacio urbano subterráneo ha sido tradicionalmente centrado en la distribución de infraestructuras, incluyendo los pasos peatonales, pero no ha tenido auge la planificación de espacios multipropósito que no solo vinculen la disposición de redes de servicios públicos y de transporte, sino también espacios para el desarrollo de diversas actividades, como si fuese una extensión del espacio público peatonal en superficie.

Como se comentó, el uso del espacio público subterráneo no ha sido aprovechado adecuadamente en la mayoría de ciudades del mundo. No obstante, hay algunas urbes que viene marcando la directriz de lo que puede lograrse. Tal es el caso de las ciudades de Arnhem y Zwolle, en Holanda. La primera, necesita desarrollar el espacio subterráneo debido que la mancha urbana llegó a sus límites y ahora el aprovechamiento del espacio subterráneo es requerimiento tanto del sector público como del privado. La segunda ciudad, es pionera en Holanda por haber incluido en su política pública de planificación al 2020, el uso del espacio subterráneo.
De manera similar, Helsinki (Finlandia) cuenta con un Plan Maestro Subterráneo que regula el uso de ese espacio en distintas categorías de acuerdo con el servicio que allí se preste. China no escapa a esta logística de planeación urbana subterránea; cerca de 20 ciudades, entre las que se cuentan Shangai y Beijing, han tenido problemas debido a que su gran y reciente crecimiento urbano no ha permitido la panificación del uso del espacio subterráneo al encontrar, por ejemplo, conflictos entre las estructura de grandes edificaciones y las redes de servicios de transporte. Debido a lo anterior, estas ciudades han planificado la coordinación del uso del espacio subterráneo pensado principalmente para estacionamientos y zonas comerciales, en integración con otros servicios como las estaciones de metro.

Un caso que expone los beneficios urbanísticos generados de un sistema subterráneo para peatones es la ciudad de Toronto. Baker [4] expone como beneficios económicos y ambientales de la infraestructura subterránea -se trata de un espacio subterráneo, desarrollado en 3 fases entre 1917 y 1984, que atiende una zona de 3.5 millones de habitantes, distribuida en un espacio de 4 x 9 manzanas. Este espacio ofrece el servicio de cerca de 1000 establecimientos comerciales, restaurantes, teatros y servicios bancarios- : incremento en el valor del suelo, independencia del tráfico peatonal y vehicular, mayor conectividad de los modos de transporte, reducción de las zonas de congestionamiento debido a la reducción de cruces vehículo / peatón, protección de los peatones ante la intemperie, mejor coordinación de las infraestructuras, y mejores propuestas paisajística para los pasos peatonales. Otra ciudad canadiense que ha desarrollado el uso multifuncional de los espacios subterráneos es Montreal, en donde estas infraestructuras permiten la circulación peatonal para protección del clima invernal que puede llegar a $-20^{\circ}$ [5]. 


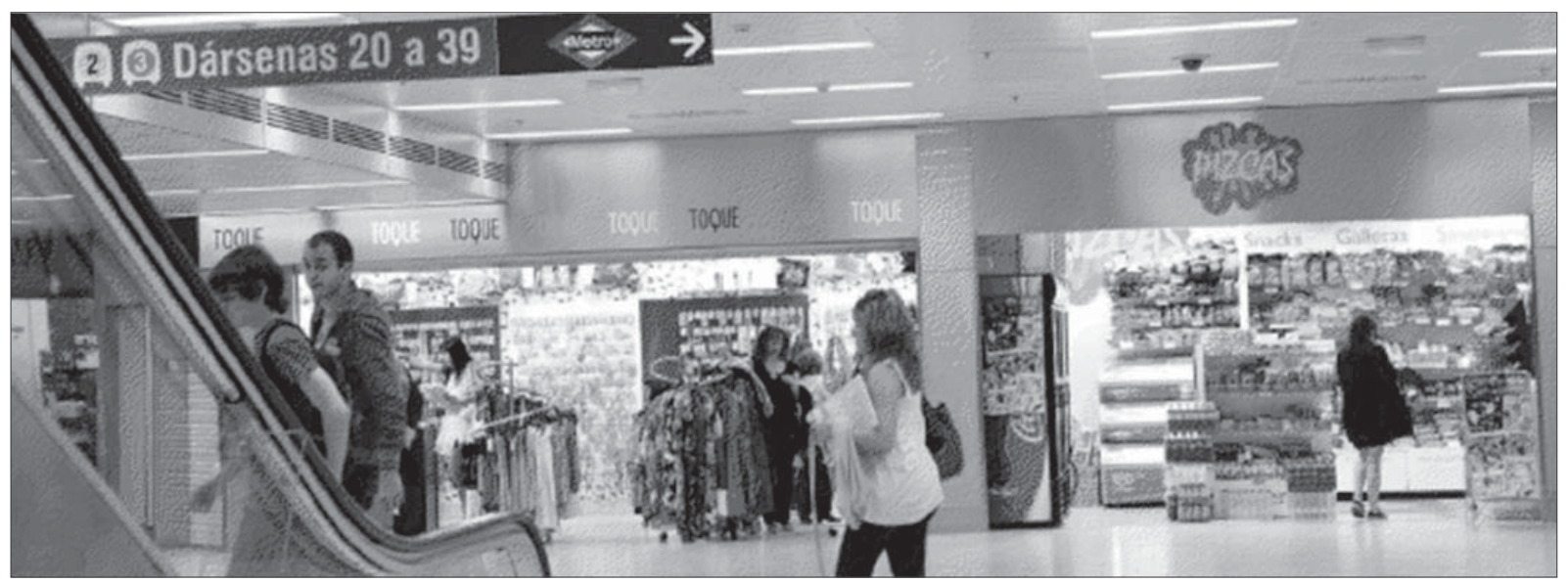

Figura 5. Pasarela subterránea que involucra conexiones de transporte público e instalaciones comerciales. Madrid

Fuente: tomado de [6]

España es otro país que se encuentra a la vanguardia en el aprovechamiento del espacio urbano subterráneo con líneas de metro, autopistas, redes de servicios, y galerías comerciales, entre otros (figura 1) [6].

Por su parte, Bogotá D.C. es un caso de ciudad en donde los desarrollos peatonales subterráneos han sido limitados. Entre ellos se cuentan dos tú- neles peatonales para cruce en vías principales: el de la calle 42 con carrera 7 (figura 2) y el túnel de la carrera 7 con calle 26 . No obstante, la escasa cuantificación de proyectos, recientemente se vienen construyendo túneles peatonales de conexión entre algunas estaciones de Transmilenio; y como proyección a mediano plazo están planificadas las futuras estaciones del Metro de Bogotá.
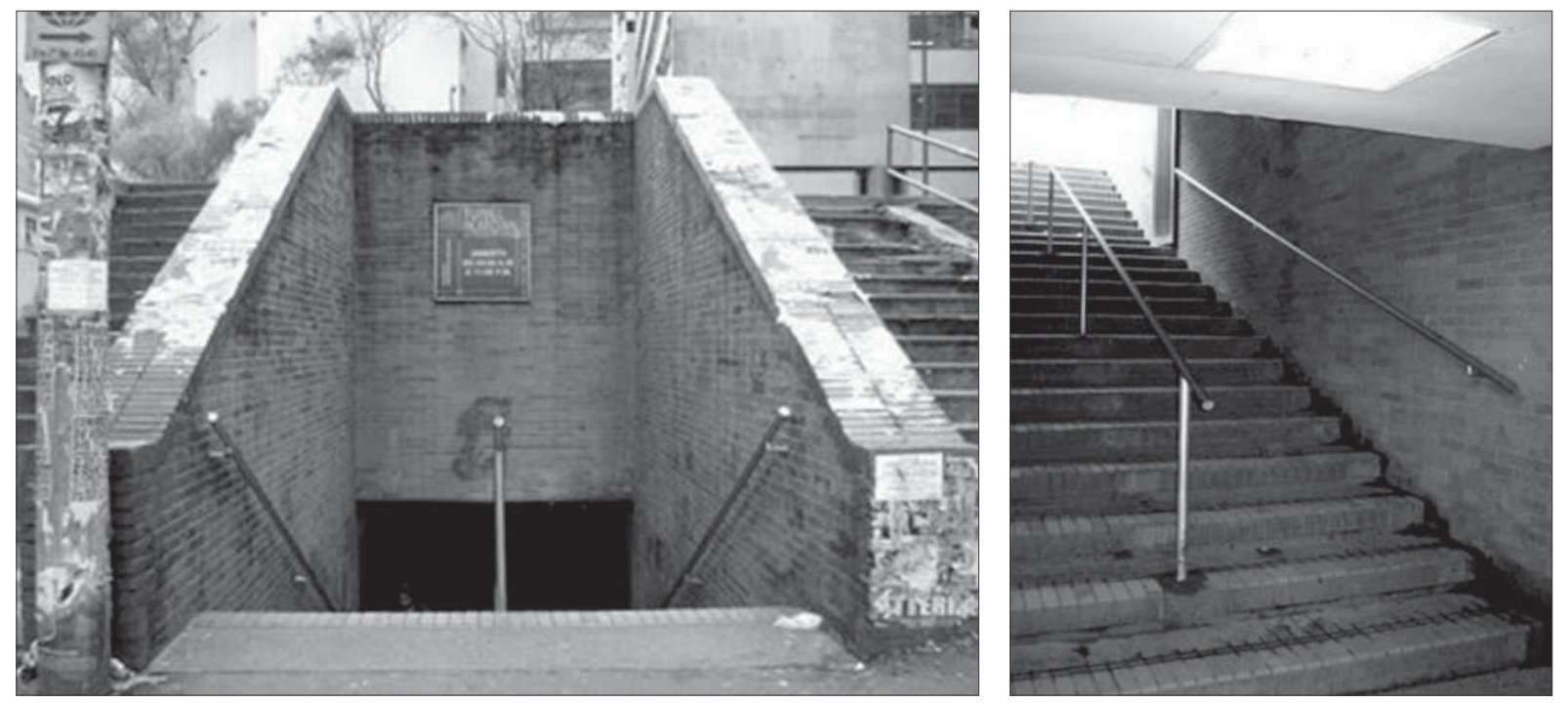

Figura 2. Accesos al túnel de la calle 42 con carrera 7. Bogotá D.C.

Fuente: disponible en: http://artespaciopublico.blogspot.com/ 
Por otro lado, cabe resaltar el hecho de que los pasos subterráneos tienden a eliminar el impacto visual generado por los pasos elevados. Estos últimos para lograr una mayor presencia visual que resulte ser una ventaja, debe resolverse con elegancia, creando un hito paisajístico en el entorno. Por el contrario, si resulta una forma rutinaria su presencia es un inconveniente, creando un entorno sin mayor atractivo [7].

\section{PREDISPOSICIÓN DEL USUARIO FRENTE AL USO DE LAS INFRAESTRUCTURAS}

Lamentablemente, diversos estudios realizados en algunos países de América Latina ponen en evidencia que los puentes peatonales no están cumpliendo su función a cabalidad, principalmente por factores que disuaden al peatón de usarlos; factores que promueven un mayor riesgo de accidentalidad vial. Si bien la mayoría de los casos documentados se orientan a los puentes peatonales, la planificación de los pasos subterráneos debe contemplar situaciones similares.

Según Hidalgo-Solorzano y otros autores [8], tras realizar una encuesta a 812 peatones de Ciudad de México, se obtuvo que cerca del $50 \%$ tiende a no usar los puentes peatonales. La principal razón fue la desmotivación por pereza $(30,7 \%)$, seguido del motivo de poder cruzar a nivel $(27,5 \%)$, y finalmente, porque consideran que los puentes son inseguros en términos de violencia y hurto $(24,8 \%)$.

En el caso de quienes sí acostumbran usar los puentes, expusieron como principal motivo la seguridad vial, seguido del hecho de que no había otra manera de cruzar la vía. No obstante, los dos motivos anteriores -del uso y el no uso de los puentes- se contraponen, pues la pereza de usar el puente expone la inseguridad vial.
Por lo anterior, se puede establecer que los peatones muestran una alta tendencia a no usar las infraestructuras de cruce a desnivel. Sin embargo, el estudio recogió las consideraciones de mejora que según los encuestados deberían aplicarse en los puentes peatonales de Ciudad de México: mayor alumbrado y seguridad (30\%), mejorar la condición de mantenimiento ( $23 \%$ ), difundir los beneficios de su uso (22\%), e impedir el paso por otra vía (12\%), entre otras recomendaciones.

En el caso colombiano, un estudio del Fondo de Prevención Vial [9], determinó que los puentes peatonales son puntos de conflicto, debido a: la falta de educación de la ciudadanía respecto al uso de los puentes, existencia de intersecciones semafóricas en cercanía a los puentes, cercanía de los puentes a centros generadores de tráfico como colegios y hospitales; y a un diseño de recorrido del puente poco atractivo para el usuario. Por su parte, la ciudad de Bogotá D.C., dispone de 272 puentes peatonales, entre los cuales se cuentan algunos que, según la ciudadanía, no son usados frecuentemente porque se duda de sus actuales condiciones estructurales. Otro aspecto que revela la falencia de los puentes peatonales en la ciudad es la sensación de inseguridad personal del usuario por hurto o atraco. Tal es el caso del puente de la calle 83 con Avenida Boyacá, del cual los usuarios frecuentes manifiestan sentir temor al usar el puente en horas de la noche.

Esos mismos criterios de disuasión al uso de puentes peatonales han sido establecidos en el caso de la ciudad de Lima (Perú), en donde de los 217 puentes peatonales de la zona, solo el $4 \%$ cuentan con techado e iluminación, un $2 \%$ solo tiene iluminación, un $11 \%$ solo está techado, y el $84 \%$ no están techados ni tienen iluminación; y adicionalmente, solo el 10\% de los puentes cuenta con rampas de acceso [10].

Un estudio realizado en el 2009 en la ciudad de Bogotá D.C. [11], en el cual se encuestaron a 225 
peatones, fue aplicado en cercanías a dos pasos peatonales subterráneos y tres elevados. El estudio concluye que hay motivos compartidos para el uso y no uso de los dos tipos de soluciones. Tanto en puentes como en túneles, su uso depende principalmente de la mayor seguridad de cruce respecto a hacerlo a nivel, y al hecho de que no hay otra alternativa para cruzar. Pero en las los motivos para no emplear los dos tipos de infraestructura, priman: el temor a la seguridad estructural del puente y a la pereza de subir las escaleras del puente.

\subsection{Percepción de pereza o cansancio res- pecto de acceder a la infraestructura}

La percepción del peatón al ver las escaleras en subida del puente es de pereza o desánimo. En el caso del túnel, primero se baja y al final del trayecto es que se toman las escaleras para subir. Este hecho propicia más al uso del túnel que del puente peatonal, por cuanto los peatones tienden a evaluar el esfuerzo físico que se requiere para cruzar la vía, y por tanto, suele preferirse bajar primero y subir después, y no la situación contraria. Este hecho se acompaña del hecho de que, generalmente, la altura a acceder en los puentes suele ser mayor que en los túneles, es decir, la subida a los puentes implica mayor tiempo y recorrido que la bajada a los túneles. Las menores longitudes de recorrido en los accesos a los túneles respecto de los puentes, se asocian también a una mayor velocidad de caminata en la infraestructura, sumado al hecho de que la velocidad peatonal bajando suele ser mayor que subiendo. En una prueba realizada en dos pasos subterráneos y tres elevados en vías principales de la ciudad de Bogotá D.C. [11], se determinó que la velocidad promedio de circulación peatonal es de $1,36 \mathrm{~m} / \mathrm{s}$ en los túneles y de $1,06 \mathrm{~m} / \mathrm{s}$ en los puentes.

\subsection{Mecanismos que obligan al uso de las infraestructuras peatonales de cruce}

Como medida para evitar la predisposición del peatón a no usar los puentes peatonales, se puede instaurar infraestructura que no permita al peatón otra alternativa de cruce distinta al puente o túnel. Un ejemplo es el cruce por puente peatonal en Vauxhall Cross (Londres), en donde el cruce por el puente (figura 3) intenta ser obligado por la presencia de barreras que impiden la continuidad del peatón (figura 4).

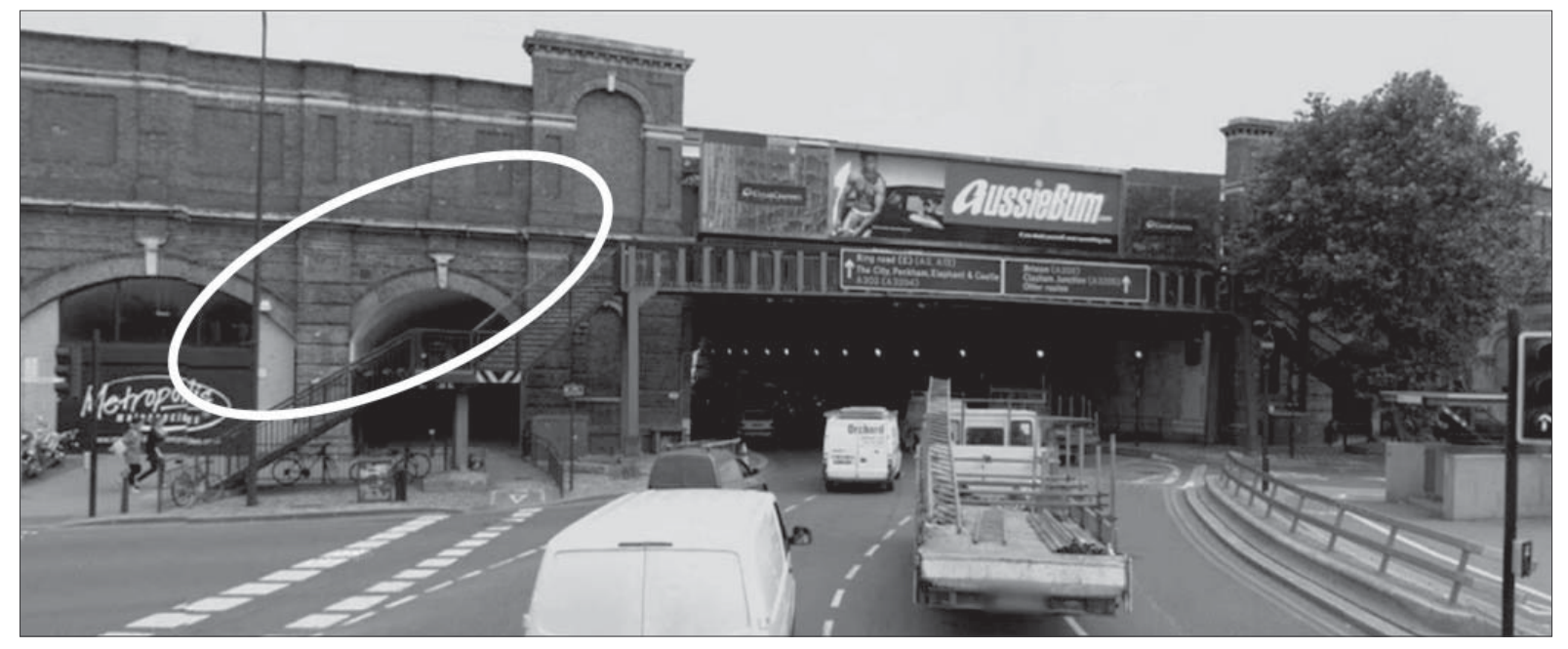

Figura 3. Cruce por puente peatonal en la intersecciónVauxhall Cross, Londres, 2011

Fuente: elaboración propia a partir de Google Earth, abril de 2011. 


\section{reflexión}

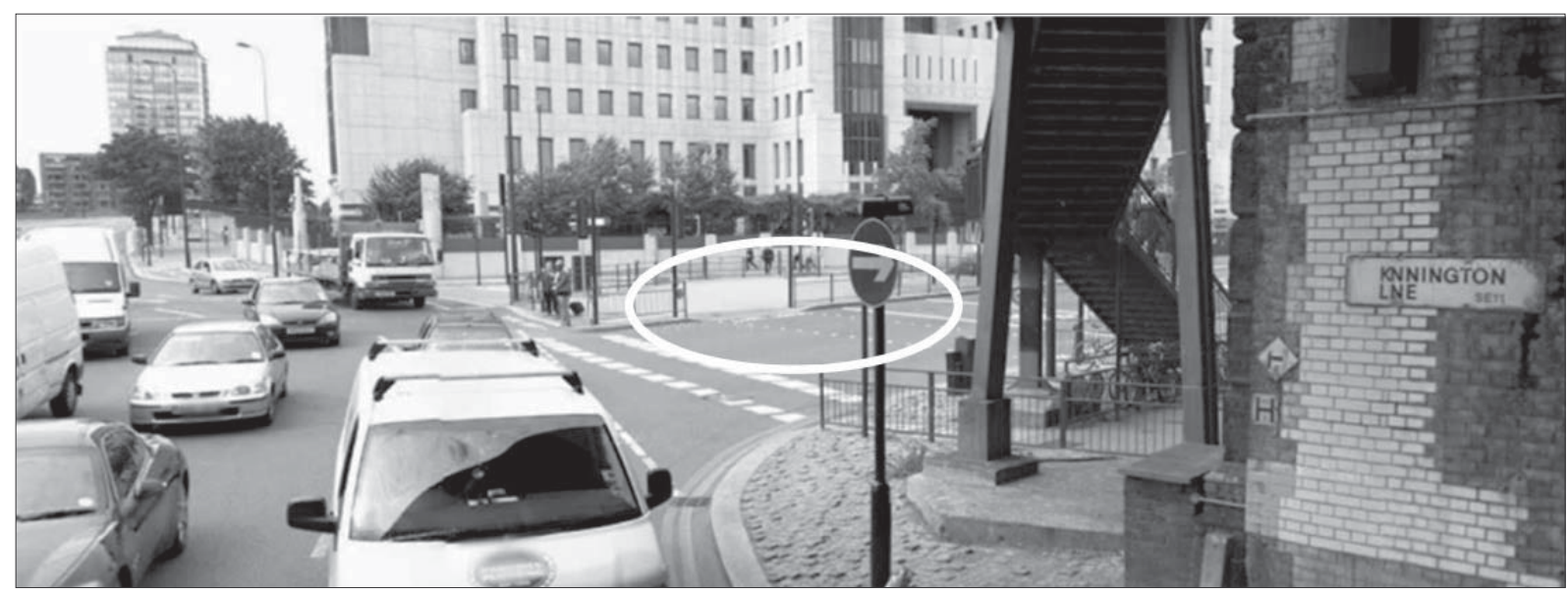

Figura 4. Cierre del paso peatonal bajo el puente de la intersecciónVauxhall Cross, Londres, 2011

Fuente: elaboración propia a partir de Google Earth, abril de 2011.

Otra opción de uso obligatorio de la infraestructura de cruce, es el uso de vallas a lo largo del eje longitudinal de la vía. Un ejemplo es la valla en new jerseys en la Carrera Séptima de Bogotá D.C. entre calles 39 y 45. Esta estructura tiene la finalidad de evitar el cruce a nivel de la vía mencionada, para que el alto flujo de estudiantes universitarios que transitan en esa zona usen el paso peatonal subterráneo de la calle 42 -mencionado anteriormente- (figura 5).

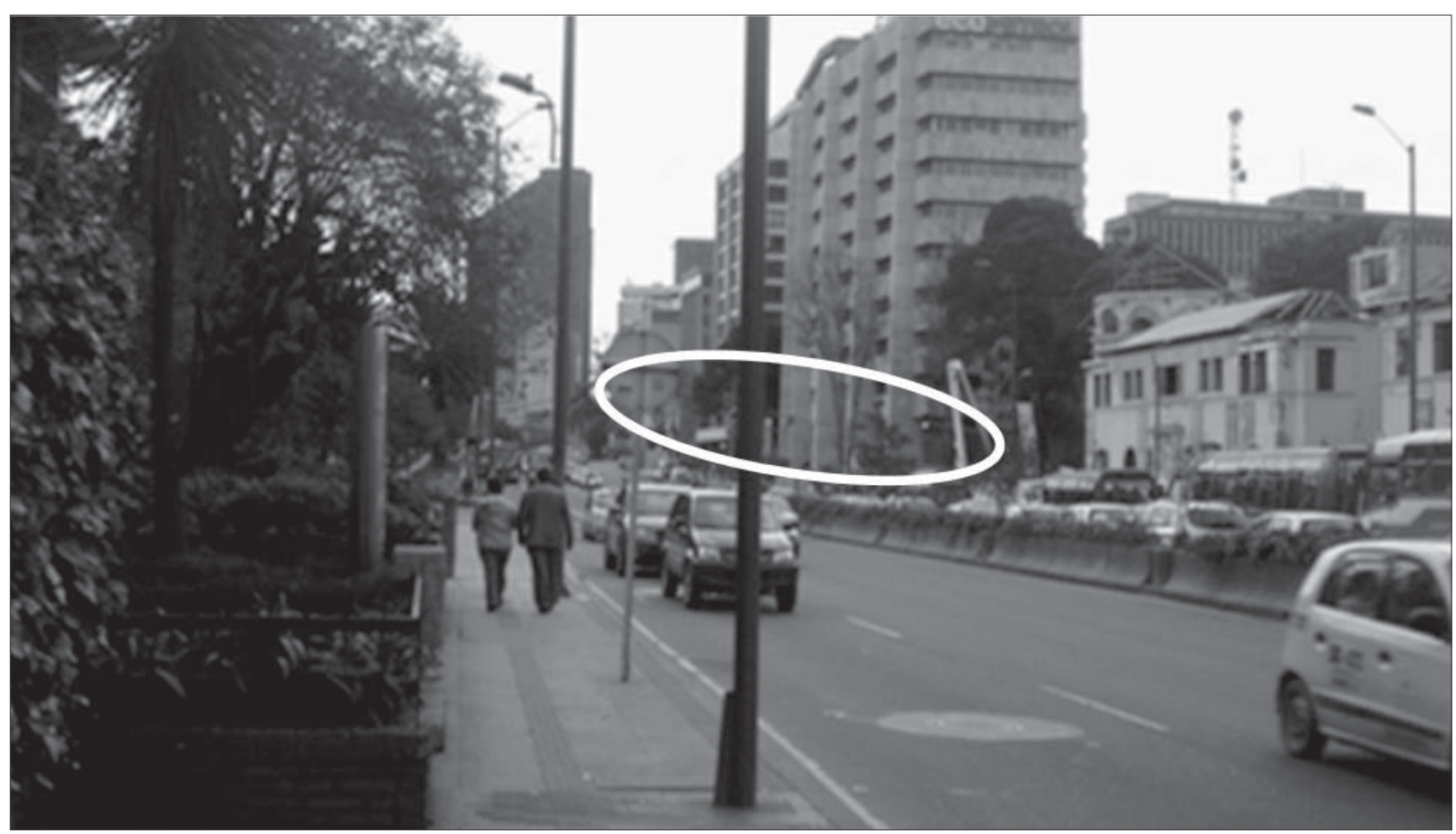

Figura 5. Valla en new jersey en la carrera séptima entre calles 39 y 45. Bogotá D.C., 2011

Fuente: elaboración propia, septiembre de 2011 


\subsection{Riesgo de accidentalidad vial para el peatón}

En el caso de Ciudad de México, Hidalgo-Solorzano [8] expone los resultados de un estudio realizado en el 2010, arrojando como características de la accidentalidad vial peatonal: la mayoría de situaciones correspondían a hombres en edad adulta productiva, y en el grupo de las mujeres la mayoría eran de edad adulta con limitaciones visuales o motoras; la mayoría de los accidentes ocurren en grandes avenidas o vías de alta velocidad, generalmente sin cruces semaforizados y con presencia de puentes peatonales. Respecto del contexto temporal, la mayoría de los atropellamientos ocurrieron en fines de semana, en horario nocturno, y en los casos que involucran hombres, estos habían ingerido bebidas alcohólicas. Una condición altamente preocupante es que, en el $90 \%$ de los casos, el conductor era una persona sin una experiencia regular al volante.

Según las estadísticas mexicanas, la población más vulnerable a la accidentalidad vial son los peatones y el $40 \%$ de las situaciones registradas en el 2005 ocurrieron en vías secundarias donde había puentes peatonales. Esto pone de manifiesto que los puentes peatonales no están cumpliendo su finalidad de infraestructura para la seguridad vial.

Una situación similar se presenta en el caso peruano de la ciudad de Lima. Según el informe: "El rol de los puentes peatonales en las vías urbanas de Lima y Callao" [10], los puentes peatonales establecidos en vías de alto flujo vehicular no están cumpliendo con los resultados esperados de evitar el cruce intempestivo e imprudente de los peatones, pues solo el $44 \%$ son utilizados frecuentemente debido a que es la única manera de cruzar, un $43 \%$ son utilizados a pesar de que también se puede hacer el cruce de vía a nivel, y un $13 \%$ son usados con poca frecuencia o no se usan debido a que la mayoría de los cruces se hacen a nivel. Como dato adicional, se tiene que en Lima el $89 \%$ de atropellos a peatón suele presentarse en cercanía a uno de los puentes de la zona.

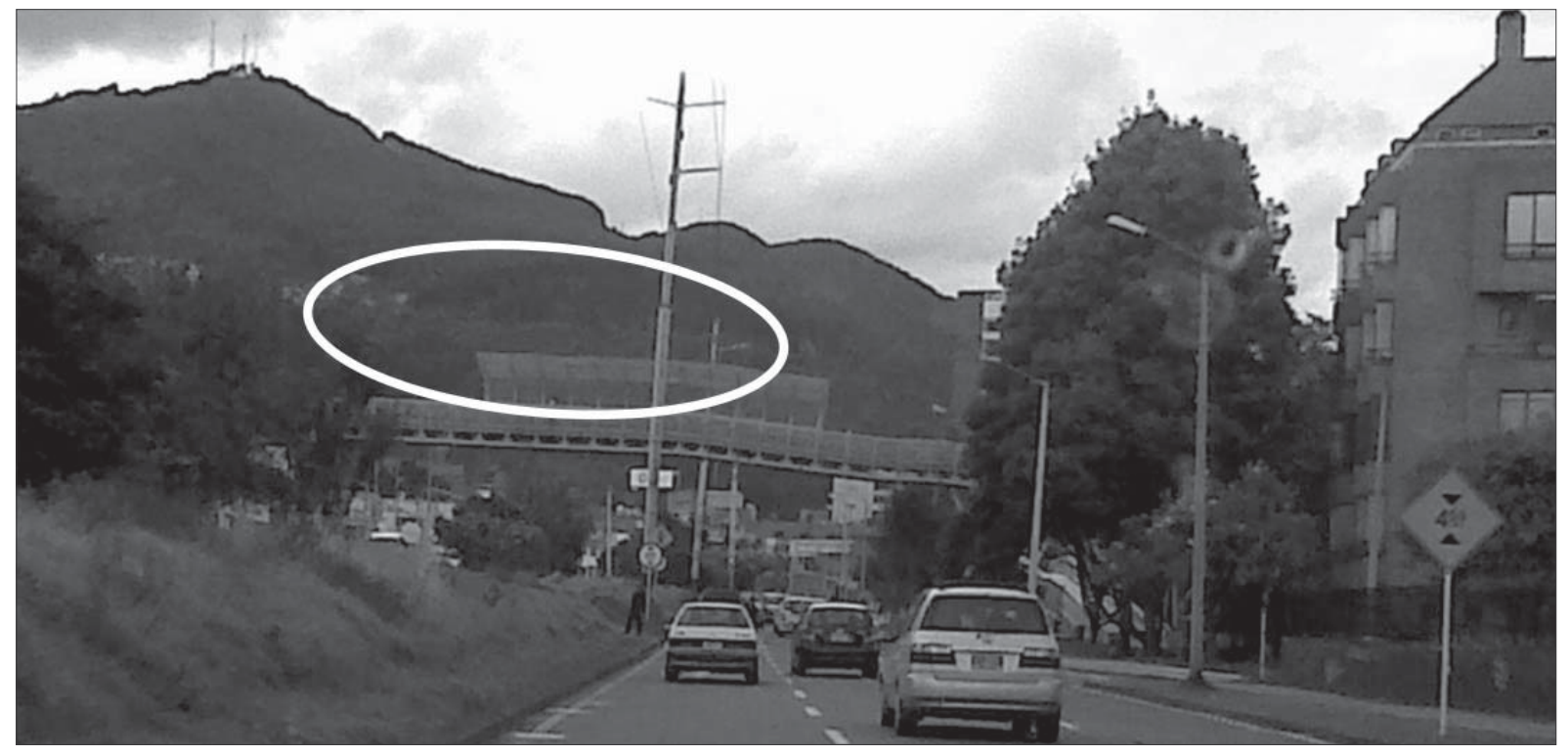

Figura 6. Puente peatonal con exposición al efecto arco de una línea de energía eléctrica de alta tensión. Carrera novena, Bogotá D.C. 2011

Fuente: elaboración propia . 
En la ciudad de Bogotá D.C., la accidentalidad vial registra cifras como: el $24 \%$ de los motivos por muertes violentas en Bogotá, en el 2007, están referidos a accidentes de tránsito, y de esa estadística, el $57 \%$ de los casos tiene al peatón como actor involucrado [12].

\subsection{Menor exposición a la intemperie}

Un factor que alienta al peatón respecto del uso de los pasos subterráneos es la menor exposición a la intemperie. Mientras en el túnel el peatón puede resguardarse de la lluvia temporalmente, o al menos mientras realiza el cruce vial; en el puente se presenta otra situación. Pero no se trata solo del resguardo de la lluvia, sino la exposición ante las emisiones de partículas contaminantes por parte de los automotores en las vías, mayores niveles de contaminación auditiva y visual. Un caso es el puente recientemente construido en la avenida carrera novena con calle $130 \mathrm{~B}$, en la ciudad de Bogotá D.C. (figura 6). Si bien la figura 6 no ilustra todas las situaciones, este puente expone al peatón no solo a la lluvia, sino a la contaminación auditiva y atmosférica causada no solo por el flujo vehicular que se supera, a un efecto mayor ocasionado por el tránsito de un tren de motricidad diesel que circula bajo el puente, y a los efectos que causa el paso de una línea de corriente eléctrica de alta tensión cuyo trazado está a pocos metros sobre el puente.

\section{MENOR OCUPACIÓN DEL ESPACIO PÚBLICO EN LOS ACCESOS}

Un análisis realizado respecto del espacio público que ocupan los accesos a pasos peatonales [13] -subterráneos y elevados-, cuantificó el área involucrada para algunos puentes y túneles peatonales en la ciudad de Bogotá D.C. (figura 7). En el caso de los accesos a los túneles peatonales -carrera séptima con calle 42 en Bogotá, carrera séptima con calle 26 en Bogotá y el acceso a la estación "Palau Reial" del Metro de Barcelona- las áreas ocupadas varían entre 10 y $50 \mathrm{~m}^{2}$, arrojando un promedio de $27 \mathrm{~m}^{2}$, aproximadamente. Por $\mathrm{su}$
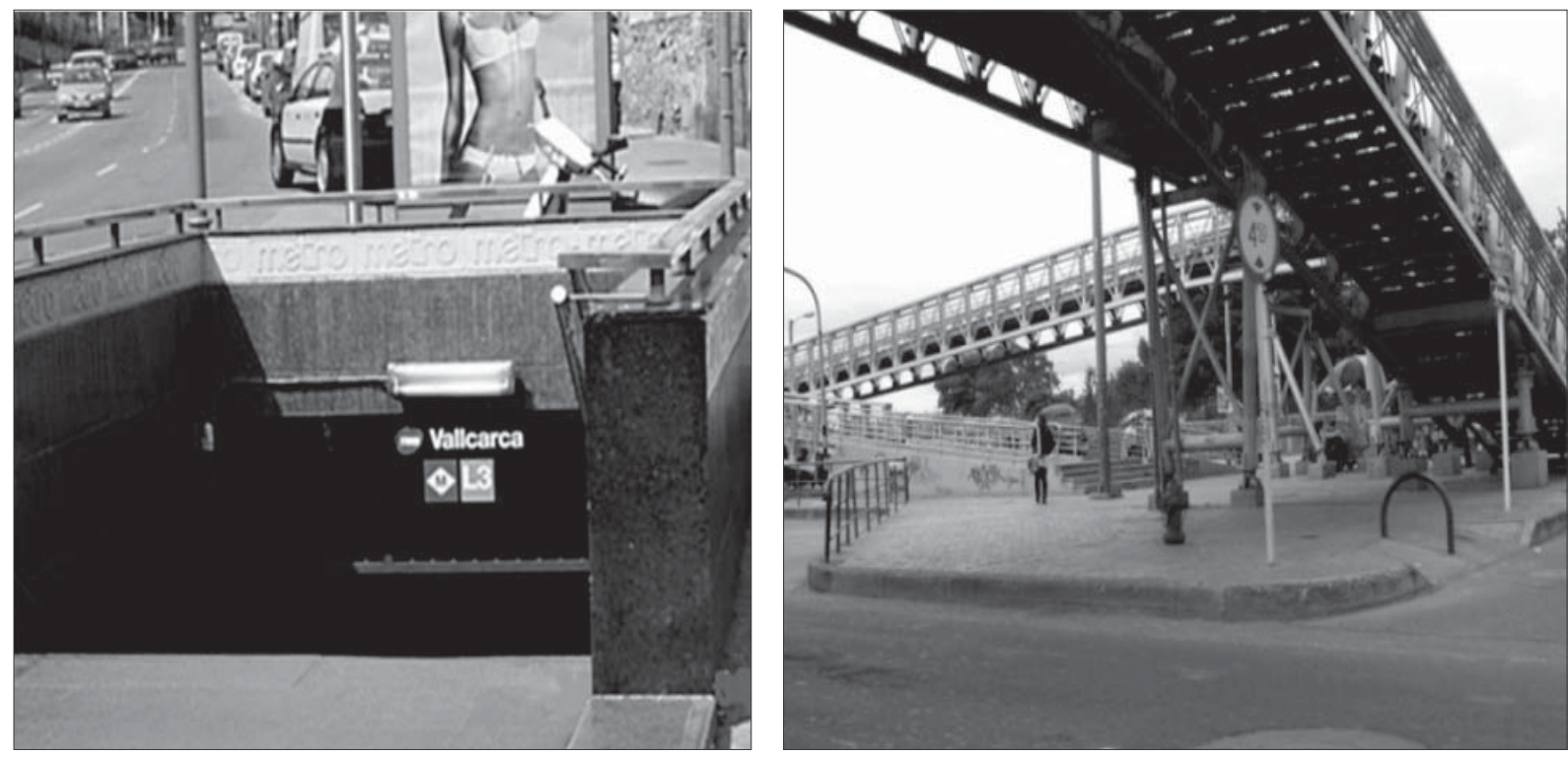

Figura 7. Acceso subterráneo a una estación de metro en Madrid (izquierda) y acceso elevado a una estación de sistema Transmilenio en Bogotá D.C. (derecha)

Fuente: tomado de [7]. 
parte, el área ocupada por los accesos a los puentes peatonales -estación "calle 142" de Transmilenio, puente de la carrera 30 con calle 57 y el puente frente al colegio Camilo Torres, todos en Bogotá- varía entre 170 y $220 \mathrm{~m}^{2}$, con un valor promedio de $225 \mathrm{~m}^{2}$.

Las cifras anteriores ponen de manifiesto que el espacio público ocupado por los accesos de los puentes -combinación de escaleras y rampasequivale a cerca de ocho veces el espacio ocupado por los accesos a los túneles. Las anteriores referencias espaciales tienen sustento en la diferencia promedio de los gálibos de los dos tipos de estructuras, que en el caso de los túneles suele estar entre 2,5 y 3 metros, y en los puentes estas cifras se duplican, llegando a valores entre 4,5 y 7 metros [7].

\section{MENORES COSTOS DE CONSTRUCCIÓN}

En nota del diario El Tiempo del pasado 25 de marzo de 2011, el Instituto de Desarrollo Urbano de Bogotá D.C.-IDU-, manifestó que los nuevos puentes peatonales metálicos que se construyen en la ciudad están teniendo un costo cercano a los \$2500 millones de pesos colombianos (COP), equivalentes a U\$ 1917808, al 12 de septiembre de 2011. Entre estos puentes se encuentran los puentes de la carrera novena con calle $130 \mathrm{~B}$ y ca1le 123, que presentan un costo de 2500 millones COP para superar un gálibo horizontal de 36 metros [14]. Lo anterior, equivale a un costo de 70 millones COP por metro lineal a superar (equivalentes a U\$ 38356, al 12 de septiembre de 2011).

Por otro lado, y de acuerdo a un estudio adelantado en la Universidad de La Salle en Bogotá D.C., en el caso de un túnel peatonal de 30 metros de longitud, construido por sistema de empuje (pipe jacking), se ha estimado un presupuesto cercano a los 1500 millones COP, lo cual equivale a un valor unitario aproximado por metro lineal de 50 millones COP (equivalentes a U\$27400, al 12 de septiembre de 2011).

Las cifras anteriores muestran que el costo de construcción de un metro lineal de puente peatonal es un $40 \%$ superior al de construcción por metro lineal de túnel peatonal.

\section{CONCLUSIONES}

Las ciudades de los países en desarrollo deben impulsar la planificación de sus esquemas de infraestructura urbana basados en principios de aprovechamiento del suelo disponible en superficie. Lo anterior se sustenta en el continuo crecimiento demográfico de las ciudades, fenómeno que tiende a desabastecer a las ciudades de suelo disponible. En ese contexto, los espacios subterráneos exponen una oportunidad para que las ciudades no detengan su crecimiento inmobiliario e infraestructural.

No obstante, cabe aclarar que las infraestructuras subterráneas por las que se propende en este artículo vinculan distintas actividades -comercio, vías peatonales, acceso a estaciones de transporte público, entre otras- en pro de la movilidad urbana peatonal. Siendo así, el escenario en que el peatón se vería más beneficiado es la seguridad vial alrededor de los cruces con vías cuyo flujo vehicular sea de gran impacto.

Pese a lo mencionado, ciudades como Bogotá D.C. siguen apostando por la solución de cruces peatonales por medio de puentes peatonales, desconociendo los beneficios que se derivan de los pasos subterráneos o túneles peatonales. Los casos expuestos en el presente artículo ponen de manifiesto diversas ventajas que presentan los primeros respecto de los segundos. Algunas de estas ventajas son: la disminución del riesgo de accidentalidad vial, menores costos de construcción 
al emplear tecnología que permita no obstruir la circulación vehicular por la vía a cruzar, mayor valía al espacio público en superficie al ocupar menores extensiones en los accesos a los túneles, menor impacto visual negativo y por tanto menores afectaciones urbanísticas desfavorables, protección ante impactos ambientales negativos, la disminución de la predisposición del usuario al cansancio por usar los túneles debido a que el primer desplazamiento es bajando y no subiendo, y a que se cuenta con menores distancias de recorrido y por tanto mayor velocidad de desplazamiento debido a menores gálibos verticales.
A pesar de las ventajas mencionadas de las soluciones subterráneas respecto de las elevadas, debe reconocerse que los dos tipos de estructuras comparten el problema de la desconfianza del peatón ante la inseguridad personal ante un posible atra$\mathrm{co}, \mathrm{y}$ la mayor facilidad que representa realizar el cruce a nivel, especialmente si el paso a desnivel está cerca de un cruce semafórico a nivel. Por este motivo, la puesta en marcha de un número cada vez más significativo de pasos peatonales a desnivel debe acompañarse de campañas de motivación y concientización de la ciudadanía frente a los beneficios de su uso.

\section{REFERENCIAS}

[1] D.S Koniohov, Uso del espacio subterráneo. Moscú, 2004. pp. 100-101.

[2] C. Torres, "Evolución histórica, actualidad y tendencia de explotación del espacio subterráneo en Bogotá - Colombia". Revista Épsilon. No. 8, pp. 57 - 70. 2007.

[3] International Tunnelling Association ITA- (2011, julio), [en línea]. Disponible en: www.ita-aites.org/index.php?id=750.

[4] M. Baker, (2011, julio), Toronto's undergound pedestrian system. Journal Tunnelling and underground space technology [en línea]. Disponible en: www. ita-aites.org/fileadmin/filemounts/general/ pdf/ItaAssociation/ProductAndPublication/WorkingGroupsPublication/WG4/ Tust_Vol_1_2_145-151.pdf.

[5] International Tunnelling Association -ITA, (2011, agosto), [en línea]. Disponible en: www.ita-aites.org/index.php?id=619.

[6] A. Pérez, A. Arteta, (2009, junio), "La vida se abre paso en el subsuelo", Revista Ca- pital. Madrid, [en línea]. Disponible en: www.ita-aites.org/fileadmin/filemounts/enews/doc/ITAnews30/capital_junio09.pdf

[7] J. González, J. Corbal y G. Capellán, "Pasarelas peatonales urbanas". Revista del Colegio de Ingenieros de Caminos, Canales y Puertos, Madrid, No. 65, pp. 70 - 77, 2003.

[8] E. Hidalgo - Solorzano, "Motivos de uso y no uso de puentes peatonales en la Ciudad de México: la perspectiva de los peatones". Revista Salud Pública en México. Vol. 52, No. 6. pp. 502-510.2010.

[9] Fondo de Prevención Vial, Determinación de puntos críticos en peatones en las ciudades del país que presentan mayores índices de accidentalidad de este actor, Bogotá D.C, 2007.

[10] Secretaría Técnica del Consejo de Transportes de Lima y Callao (2008), El rol de los puentes peatonales en las vías urbanas de Lima y Callao, [en línea]. Disponible en: http://www.ctlc-st.gob. 
pe/2008/Puentes\%20Peatonales/EL\%20 ROL\%20DE\%20LOS\%20PUENTES\%20 PEATONALES\%20EN\%20LAS\%20 V\%C $3 \% 8$ DAS \%20URBANAS $\% 20$ DE\%20LIMA\%20Y\%20CALLAO.pdf.

[11] L. Castañeda, C. Torres, Evaluación comparativa de los pasos peatonales elevados y subterráneos para Bogotá, Universidad de La Salle, 2010.

[12] Cámara de comercio de Bogotá, Boletín de accidentalidad vial, No. 2, 2008.
[13] C. Urazán, H. Rondón, "Relación entre el espacio público y la infraestructura de un sistema de transporte masivo. Caso Transmilenio en Bogotá", Revista Studiositas, Vol. 5, No. 2. pp. 37-48. 2010.

[14] Diario el Tiempo (26 de julio de 2011), Tras un año de retraso, habilitado puente peatonal de la 182 con séptima. Bogotá D.C., [en línea]. Disponible en: http:// www.eltiempo.com/archivo/documento/ CMS-10010765. 